Technical editor: Jackie Annis jannis@ bmj.com (+44 (0)20 7383 6658)

Please note: This paper contains visible codes for electronic publishing. These will not appear in the final version and alignment of tables will be improved.

- Please check that all queries to author [in bold type and square brackets] have been answered, including those in the references and tables. Please do not renumber the references. Any additional references can be added to the bottom of the list and unwanted references deleted from the list without renumbering. The numbering will be corrected automatically during processing

\title{
Using patient data for patients' benefit
}

Full partnership will help ensure that data really does save lives

Amitava Banerjee, ${ }^{1}$ senior clinical lecturer, David Mathew, ${ }^{2}$ patient and public panel member, Katherine Rouane, ${ }^{3}$ patient

${ }^{1}$ University College London, London, UK

${ }^{2}$ University of Bedfordshire, Luton, UK

3 London

Correspondence to: A Banerjee ami.banerjee@ucl.ac.uk

\section{2}

The \#datasaveslives campaign underlines the importance of health informatics research to public health. ${ }^{1}$ There are many examples of "data driven" healthcare across the UK and elsewhere, such as increased use of machine learning in early diagnosis of tumours and automated risk prediction tools for cardiovascular disease built into electronic health records (EHR). Innovations are often labelled as "personalised" and "patient centred,"2-4 but since all health data are personal and about patients, what does the terminology actually mean, and do these innovations benefit patients?

Routine healthcare data worldwide are designed for delivering care. Information from patients or health professionals may be recorded in written, spoken, or electronic formats, depending on the setting. Electronic health data come from a diverse range of sources, including health management information systems, disease surveillance, registries, hospital coding systems, pharmacies, laboratories, questionnaires, government coding systems, insurance claims databases, social media platforms, and other third parties such as mobile phone companies.

From banking and online shopping to booking holidays, other sectors are well ahead of healthcare in their use of technology and routine data for the benefit of the end user. The Wachter review of NHS information technology recommended better links between local and national health data to improve standards of care, as did the Caldicott review, with the proviso of improved public trust in data security. ${ }^{56}$ 
Worldwide however, government policy initiatives have focused largely on "paperless" records and cybersecurity, with little mention of the use of data for the direct benefit of patients . Patient benefit can be variably defined by research evidence of better outcomes, added value, quality improvement, increased safety, enhanced patient experience, or a combination of these. Add in the heterogeneity of routine health data, and the magnitude of the task becomes more apparent.

Much of the international discourse and media coverage around health data hinges around data security, privacy, and regulations. For example, in Europe, the General Data Protection Regulation intends to strengthen and unify data protection for individuals, committing organisations involved in healthcare to be transparent about how they use data. ${ }^{7}$ Pressure is on hospitals and universities to optimise their use of routine data, but patients will be empowered only if they are involved in defining "patient centredness," a term often contrasting with their experiences as faceless hospital numbers in a busy clinic or on a crowded ward.

Some patients do not want their information electronically gathered or stored because of lack of clarity about future use and concerns about security (magnified by the recent NHS cyberattack). To many the digital world, social media campaigns, and hashtags are meaningless, intimidating, and a barrier to realistic engagement. ${ }^{8}$ When patients neither know nor understand, they are unlikely to consent to use of their data. However, if patient benefit is explained, many will be happy to cooperate and expectations of risk, benefit and harm will be better managed.

As uptake of electronic health records increases, there are many opportunities to enhance use of routine healthcare data. Real time application in clinical decision making will improve quality of care, including through development of better risk prediction models, better monitoring of patient outcomes, and the "right data for the right patient at the right time." Use of routine data can also reshape health and social care research by reducing $\operatorname{costs}^{10}$, increasing generalisability (by incorporating real world populations), and boosting the translation of research into practice. In the UK, this vision is reflected in several strategic publications by government, research councils, and charities. ${ }^{5,6,11,12}$

If efforts in many health systems to improve interoperability and to link data across sources and sectors are successful, ${ }^{10}$ there is unprecedented scope to build "learning health systems. ${ }^{13}$ These integrate and facilitate data capture and use across research, evidence based guidelines, and clinical care, ultimately increasing the value of routine data for individual patients. 
Proper involvement and engagement of patients in the whole process, including access to data and user centred design of systems and research, has the potential to change the culture of data usage in healthcare, to reduce waste and redundancy, and to rebuild trust. ${ }^{4} 1415$ Finally, the optimism of data-driven care, whether in precision medicine or clinical decision support tools must be evidence-based and that evidence should be patient-centred.

The most patient-centred approach to data usage is to make best use of available data in a way that patients and the public want and expect. However, there seems to be a mismatch between clinicians and patients in the perception of benefit. A change in culture, and a clear strategy of patient engagement is necessary to reflect the beliefs and values of patients without creating an excessive burden for patients or clinicians, and ultimately to ensure that data really does save lives.

This editorial is the output from a workshop which $\mathrm{AB}$ conceived and facilitated at the BMJ Evidence Live Conference, Oxford 2017. In addition, expert consensus and reaction was sought by AB at the Chief Clinical Informatics Officer (CCIO) Summer School, Manchester 2017. DM and KR are on the patient and public engagement panel of the Farr Institute of Informatics Research, London

Competing interests: We have read and understood BMJ policy on declaration of interests and have no relevant interests to declare.

Provenance and peer review: Commissioned; not externally peer reviewed.

<eref>1. Farr Institute of Health Informatics Research. \#datasaveslives. http://www.farrinstitute.org/public-engagement-involvement/datasaveslives

<eref>2 National Information Board and Department of Health. Personalised health and care 2020. 13/11/2014.https://www.gov.uk/government/publications/personalised-healthand-care-2020</eref>

<jrn>3 Velardo C, Shah SA, Gibson O, et al; EDGE COPD Team. Digital health system for personalised COPD long-term management. BMC Med Inform Decis Mak 2017;17:19. PubMed doi:10.1186/s12911-017-0414-8</jrn>

<jrn>4 Biswas R, Martin CM, Sturmberg J, et al. User-driven health care-answering multidimensional information needs in individual patients utilizing post-EBM approaches: a conceptual model. J Eval Clin Pract 2008;14:742-9. PubMed doi:10.1111/j.13652753.2008.00998.x </jrn>

<eref>5 Department of Health. Making IT work: harnessing the power of health information technology to improve care in England. 2016. https://www.gov.uk/government/uploads/system/uploads/attachment_data/file/550866/Wacht er_Review_Accessible.pdf $</$ eref $>$ 
<eref>6 National Data Guardian for Health and Care. Review of data security, consent and opt-outs. 2016.https://www.gov.uk/government/publications/review-of-data-securityconsent-and-opt-outs $</$ eref $>$

<eref>7 Information Commissioner's Office. Overview of the General Data Protection Regulation (GDPR). https://ico.org.uk/for-organisations/data-protection-reform/overview-ofthe-gdpr/</eref $>$

<jrn>8 Latulippe K, Hamel C, Giroux D. Social health inequalities and ehealth: a literature review with qualitative synthesis of theoretical and empirical studies. J Med Internet Res 2017;19:e136. PubMed doi:10.2196/jmir.6731</jrn>

$<$ jrn>9 Davis S, Roudsari A, Courtney KL. Designing personal health record technology for shared decision making. Stud Health Technol Inform 2017;234:75-80. PubMed</jrn>

$<$ jrn>10 Sheikh A, Sood HS, Bates DW. Leveraging health information technology to achieve the "triple aim" of healthcare reform. J Am Med Inform Assoc 2015;22:849-56. PubMed doi:10.1093/jamia/ocv022</jrn>

<eref> 11 Medical Research Council UK. Maximising the value of UK population cohorts MRC strategic Review of the largest UK Population cohort studies. 2014. https://www.mrc.ac.uk/publications/browse/maximising-the-value-of-uk-population-cohorts/

<eref> 12 Public Health Research Data Forum. Sharing research data to improve public health: full joint statement by funders of health research. 2010. https://wellcome.ac.uk/what-we-do/our-work/sharing-research-data-improve-public-healthfull-joint-statement-funders-health

<edb>13 Olsen LA, Aisner D, McGinnis JM, eds. The learning healthcare system: workshop summary. The national academies collection: reports funded by National Institutes of Health. 2007.</edb>

$<$ jrn>14 Ziebland S, Powell J, Briggs P, et al. Examining the role of patients' experiences as a resource for choice and decision-making in health care: a creative, interdisciplinary mixed-method study in digital health. Programme Grants for Applied Research 2016;4. PubMed</jrn>

<jrn>15 Santana MJ, Haverman L, Absolom K, et al. Training clinicians in how to use patient-reported outcome measures in routine clinical practice. Qual Life Res 2015;24:170718. PubMed doi:10.1007/s11136-014-0903-5</jrn> 